\title{
Eficacia de un programa de psicoestimulación integral en pacientes con diagnóstico de enfermedad de Alzheimer
}

\author{
María Burillo $^{1}$, Sonia Salvador ${ }^{1}$, Marina García ${ }^{1}$, Marina Romance ${ }^{1}$, \\ Remedios Labar ${ }^{1}$, Laura Calvera ${ }^{2}$, Cristina Soler ${ }^{1}$, Gemma Cordero ${ }^{3}$, \\ Laura Quijada $^{1}$, y Marta Mota ${ }^{1}$ \\ ${ }^{1}$ Hospital Universitario Miguel Servet (HUMS), Zaragoza (España); \\ ${ }^{2}$ Hospital San Jorge, Huesca (España); ${ }^{3}$ Centro de Rehabilitación Psicosocial \\ Nuestra Señora del Pilar, Zaragoza (España)
}

\begin{abstract}
La enfermedad de Alzheimer se define por un cuadro de demencia, es decir, por un proceso progresivo de deterioro cognitivo, y funcional de inicio insidioso al que se pueden asociar alteraciones conductuales. La propuesta de intervención que se plantea es un programa de psicoestimulación integral que se compone de diferentes estrategias terapéuticas no farmacológicas. El objetivo del trabajo es, evaluar la eficacia de un programa de psicoestimulación integral tanto en el mantenimiento del rendimiento cognitivo como en la mejoría de la calidad de vida en pacientes recientemente diagnosticado de enfermedad de alzheimer y que presentan un deterioro cognitivo leve- moderado. La muestra se compuso de ocho personas. Los instrumentos utilizados fueron la escala de Mini-Examen Cognoscitivo y la escala Quaity of Life in a Disease Scale. Los resultados indicaron a nivel cognitivo, discretas mejorías. El 50\% de los pacientes obtienen la misma puntuación que al inicio, el 37,5\% obtuvo una mejoría. En relación a la calidad de vida, el 50\% de los pacientes mejoran, el 12,5\% mantiene la misma nota, sin embargo, 37,5\% empeoran. En conclusión, los hallazgos de este estudio revelan que a pesar de que la intervención no produjo mejorías significativas en las variables medidas, los pacientes referían una mejoría clínica cualitativa principalmente a nivel funcional.
\end{abstract}

Palabras clave: Enfermedad de alzheimer, psicoestimulación cognitiva, calidad de vida, rendimiento cognitivo.

Efficacy of a comprehensive psychostimulation program in patients diagnosed with Alzheimer's disease. Alzheimer's disease is defined by a picture of dementia, that is, by a progressive process of cognitive impairment, and insidious functional onset to which behavioral alterations can be associated. The proposed intervention is a comprehensive psychostimulation program that consists of different non-pharmacological therapeutic strategies. The objective of the work is to evaluate the efficacy of a program of integral psychostimulation both in the maintenance of cognitive performance and in the improvement of the quality of life in patients recently diagnosed with Alzheimer's disease and who have mild-moderate cognitive impairment. The sample consisted of eight people. The instruments used were the Cognitive Mini- Examination scale and the Quaity of Life in a Disease Scale. The results indicated cognitively, slight improvements. $50 \%$ of patients get the same score as at the beginning, $37.5 \%$ obtained an improvement. In relation to quality of life, $50 \%$ of patients improve, $12.5 \%$ maintain the same score, and however, $37.5 \%$ get worse. In conclusion, the findings of this study reveal that although the intervention did not produce significant improvements in the measured variables, the patients reported a qualitative clinical improvement mainly at the functional level.

Keywords: Alzheimer's disease, cognitive psychostimulation, quality of life, cognitive performance.

Correspondencia: María Burillo Gonzalvo. Hospital Universitario Miguel Servet (HUMS). Avenida San José, nº 121. C.P.: 50008. Zaragoza (España). E-mail: maria.burillo@ hotmail.es 
La enfermedad de Alzheimer (EA) es una entidad clínica, caracterizada por un cuadro de demencia, esto es, por un proceso progresivo de deterioro cognitivo y funcional de inicio insidioso al que en el transcurso del tiempo se pueden asociar cambios en el estado de ánimo, alteraciones conductuales y psicóticas. Las alteraciones neuropatológicas que la provocan se sitúan fundamentalmente en la corteza cerebral, sobre todo en las áreas temporoparietales, donde se puede observar la presencia de signos anatomopatólogicos característicos: placas seniles o neuríticas, placas amiloideas, pérdida neuronal y ovillos neurofibrilares (Villar, Molinuevo, y Gómez, 2004).

La EA se caracteriza por su heterogeneidad, tanto clínica como etiológica. Asimismo, el diagnóstico se basa en la aplicación de criterios clínicos, dado que no existen pruebas biológicas específicas para ello. En estos criterios, para establecer el diagnóstico definido se requiere de la comprobación histopatológica, mientras que el diagnóstico de EA probable puede realizarse si existe una demencia de inicio insidioso y curso progresivo en ausencia de otras enfermedades cerebrales que puedan explicar el trastorno cognitivo. Si existen otras enfermedades que pueden justificar el deterioro (como, por ejemplo, infartos cerebrales, cífilis, etc.), pero la EA se considera la principal causante, entonces el diagnóstico será de EA posible (Villar et al., 2004).

Existe un gran número de estudios de prevalencia sobre las demencias en población europea y todos ellos señalan que, con la edad, la prevalencia de la EA se incrementa. En concreto, el estudio EUROMODEM, mostró que el 53,7\% de los casos de demencia correspondían a la EA, lo que conlleva una prevalencia del 4,4\%. La prevalencia española de la EA, se estableció en el 5,6\% en la población de 75 años o más (Virués et al., 2011).

En la EA la patogénesis es desconocida, si bien se considera una enfermedad de causalidad multifactorial, siendo el factor de riesgo más importante la edad. La clínica de la EA es muy variable y se puede agrupar en tres áreas: cognitiva, funcional y conductual. Para que pueda realizarse el diagnóstico de demencia, los déficits cognitivos y conductuales deben suponer una afectación de las actividades de la vida diaria del paciente. Entre el 60 y el 70\% de los pacientes siguen un patrón evolutivo típico. El patrón neuropsicológico inicial de una EA típica se define por una afectación de la memoria episódica verbal y visual junto con alteraciones de la denominación y alteraciones incipientes de la memoria semántica. A medida que la enfermedad avanza, aparecen alteraciones de las praxias constructivas e ideomotoras por imitación, junto con alteraciones ejecutivas frontales y de la percepción visual compleja (Peña, 2007).

El síntoma más frecuente en el área conductual es la apatía, pero también cursa con otros síntomas afectivos y conductuales, como alucinaciones auditivas o visuales e ideas delirantes, generalmente de robo o infidelidad. Otros son agitación psicomotriz, irritabilidad y desinhibición. Existe, también, un declive progresivo en la actividad funcional del paciente, por lo que las actividades de la vida diaria (AVD) se irán mermando. Inicialmente, se afectan las actividades avanzadas, como la actividad laboral 
o las relaciones sociales, para posteriormente, deteriorarse las AVD instrumentales, como el cuidado del hogar o el manejo del dinero. Finalmente, se deterioran las AVD básicas, como la alimentación, la higiene, o el control de los esfínteres. Habitualmente, el paciente progresa a un estado vegetativo y fallece por otra enfermedad intercurrente. Como ya se ha comentado, en la EA las alteraciones cognitivas, perceptivas, motoras y conductuales repercuten en el funcionamiento del paciente y en su calidad de vida y éstas se ven gravemente mermadas conforme avanza la enfermedad (Alberca y López, 2011).

A causa del envejecimiento continuo de la demografía, la incidencia de la demencia se incrementará de forma vertiginosa, siendo previsible a nivel mundial, una auténtica epidemia. Este cambio supondrá un coste económico, sanitario y social muy importante, que principalmente recaerá en los familiares, puesto que son los cuidadores principales del enfermo. El tratamiento de la demencia, especialmente la EA, hasta el presente se ha focalizado en la administración de fármacos para el manejo de la clínica vinculada a la enfermedad. Pero dicha estrategia terapéutica se ha considerado insuficiente puesto que se requiere un abordaje integral, también a través de terapias no farmacológicas (TNF). En la última década autores como Olazarán, Poveda, Martínez, y Muñiz (2007) han planteado la importancia de establecer una formula dinámica entre ambas utilizando aquellos tratamientos que evidencien seguridad y eficacia.

El concepto de TNF abarca todo un conjunto de métodos y estrategias terapéuticas que pretenden mantener preservadas las funciones cognitivas y mejorar la autonomía de los pacientes y de este modo, potenciar su calidad de vida. Se han propuesto múltiples TNF para el tratamiento de las demencias: dirigidas al entrenamiento de las funciones cognitivas, otras a paliar los trastornos de conducta, otras a través de técnicas de restructuración ambiental con el objetivo de disminuir la dependencia del paciente y finalmente también dirigidas al ámbito familiar. Es fundamental establecer los objetivos a alcanzar con cada intervención, así como evaluar su repercusión en otras áreas dado que cada intervención puede influir sobre otras, generalmente positivamente, pero también podría tener un efecto negativo (García y Carro, 2011).

En la actualidad, a pesar de que existen múltiples trabajos en este campo, todavía existen pocos datos sobre su eficacia real, principalmente por problemas metodológicos como la propia heterogeneidad de la enfermedad, la dificultad de estandarizar y comparar resultados con protocolos de intervención grupal, la elección de parámetros de eficacia adecuados o las dificultades en el diseño de estudios multicéntrico con amplia muestra (Francés, Barandiarán, Marcellán, y Moreno, 2003).

De los estudios publicados, Tárraga (1991), en los años noventa, afirma que, en un $75 \%$ de los casos de demencia leve-moderada este tipo de terapias pueden utilizarse como estrategia terapéutica y esto puede ser beneficioso tanto para el paciente como para la familia. Posteriormente, se concretará que la estimulación cognitiva y las terapias de 
orientación a la realidad son las terapias no farmacológicas más utilizadas y eficaces (Olazarán et al., 2004).

Olazarán et al. (2010) publican una revisión sistemática sobre la eficacia de las TNF en la EA y concluye que se afianzan como estrategias tanto eficaces como eficientes, para mejorar las manifestaciones clínicas y la calidad de vida tanto de las personas con demencia como del cuidador. En concreto, obtienen recomendación grado B las intervenciones multicomponente, compuestas por estimulación cognitiva más alguno de los siguientes integrantes: reminiscencia, ejercicio físico, entrenamiento en AVD y/o apoyo. Se observa tras su implementación mejoría en las siguientes áreas de las personas con demencia; cognición, AVD, conducta, estado de ánimo y calidad de vida.

En términos generales, la intervención debe atender a todos los componentes de la persona, desde los cognitivos, hasta los emocionales, pasando por el ámbito del comportamiento. Su aplicación será flexible y personalizada. En relación a las habilidades del terapeuta, es necesario establecer con los pacientes una relación forjada por la calidez, la validación emocional y la empatía. Además, dicha intervención se debe situar en el contexto general del tratamiento de la persona. Franco y Orihuela (1998) señalan que los programas de psicoestimulación y de rehabilitación neuropsicológica, deben cumplir los siguientes requisitos para conseguir utilidad clínica; ser dinámicos, flexibles, sencillos, económicos, fácilmente disponibles y útiles.

En este trabajo, la propuesta de intervención que se plantea está dirigida a un grupo de ocho personas recientemente diagnosticadas de EA. Consiste en un programa de psicoestimulación integral que se compone de diferentes estrategias terapéuticas no farmacológicas. Se fundamenta en la presencia de neuroplasticidad cerebral, dicho de otra forma, la reacción del cerebro para adaptarse a las situaciones novedosas para restablecer el equilibrio alterado, basándose tanto en la neuropsicología cognitiva, como en las terapias de modificación de conducta. Así pues, la finalidad principal de las terapias de psicoestimulación es la de potenciar la neuroplasticidad por medio de la muestra de estímulos, estudiados previamente, que promuevan de forma integral las capacidades emocionales, intelectuales, físicas y relacionales.

El objetivo general de la intervención es determinar pautas para mantener las capacidades cognitivas preservadas, mejorar la funcionalidad y autonomía en las actividades de la vida diaria, y de esta forma, aumentar la calidad de vida de los pacientes y de sus familiares.

Los objetivos específicos son: estimular las capacidades cognitivas, incrementar la autonomía personal, mejorar la gestión del comportamiento; estimular la propia identidad y la autoestima, favorecer la socialización, mejorar el estado de salud, reducir la carga familiar, y aumentar la calidad de vida del paciente y de sus familiares.

El propósito del presente trabajo es, por un lado, exponer un programa de psicoestimulación integral compuesto de diferentes estrategias no farmacológicas dirigido 
a pacientes recientemente diagnosticados de EA y por otro, evaluar su eficacia tanto en el mantenimiento de su rendimiento cognitivo como en la mejoría de su calidad de vida.

\section{MÉTODO}

\section{Participantes}

La muestra está compuesta por ocho personas, de los cuales tres son hombres y cinco son mujeres. Todos ellos han sido recientemente diagnosticados de EA y presentan un deterioro cognitivo leve-moderado, con una calificación de tres-cuatro en la Escala de Deterioro global de Reisberg (GDS). Su media de edad es de 76 años y su escolaridad es de estudios mínimos, lecto-escritura básica.

\section{Variables e instrumentos de medida}

Las variables e instrumentos de medida empleados para valorar la eficacia del programa se enumeran a continuación:

-Evaluación del estado cognitivo: Se administró la escala de Mini-Examen Cognoscitivo (MEC) (Lobo et al., 1999), la cual realiza un cribado del déficit cognitivo. Se compone de veintidós ítems y las áreas cognitivas que evalúa son la orientación espacial y temporal, atención y cálculo, memoria inmediata, lenguaje y memoria diferida.

-Evaluación de la calidad de vida: Se aplicó la escala Quaity of Life in a Disease Scale (QOL-AD) (Hoe, Katona, Roch, y Livingston, 2005) la cual se compone de trece ítems y valora trece dimensiones: energía, salud física, estado de ánimo, condiciones de vida, familia, memoria, vida social, relación personal estrecha, visión general de sí mismo, capacidad para realizar tareas de casa, situación financiera, la vida en general y capacidad para hacer cosas por diversión.

\section{Recogida de datos}

En primer lugar, se valoró el deterioro cognitivo del paciente. La recogida de datos se realizó en dos ocasiones mediante la escala de valoración MEC (Lobo et al., 1999). La primera, se realizó antes de comenzar la intervención y la segunda, se efectuó al finalizar la intervención. En el caso de la calidad de vida, la recogida de datos se desarrolló en dos ocasiones al comenzar la intervención y al finalizarla. Para su evaluación se utilizó la escala de valoración QOL-AD. Estos datos sirvieron de referencia para determinar la efectividad de la intervención.

\section{Procedimiento}

El programa de psicoestimulación integral consiste en actividades individuales tuteladas y personalizadas para cada paciente, así como actividades grupales. Los tres bloques fundamentales de la misma son; la terapia de orientación a la realidad, la 
psicoestimulación cognitiva y la actividad grupal (Tabla 1). Este se realizará semanalmente con una duración de una hora y treinta minutos y un total de tres meses (doce sesiones).

Tabla 1. Estructura de las sesiones

\begin{tabular}{lc}
\hline Duración & Estructura de las sesiones \\
\hline 5 minutos & Bienvenida \\
\hline 5 minutos & Terapia de Orientación a la Realidad \\
\hline 40 minutos & Psicoestimulación Cognitiva \\
\hline 35 minutos & Actividad Grupal \\
\hline 5 minutos & Cierre de la Sesión \\
\hline
\end{tabular}

\section{Actividades}

A continuación, se presentan específicamente cada una de las actividades planteadas.

Bienvenida: Se realiza al inicio de cada sesión. En este primer momento se saludará al grupo y se les preguntará sobre cómo se encuentran y sobre cómo les ha ido la semana. Además, se les explicará brevemente cuáles serán las actividades que se realizarán durante la sesión.

Terapia de Orientación a la realidad: Los objetivos de esta actividad son: favorecer y activar la orientación en las tres esferas (espacio, tiempo y persona), conservar la conexión con el entorno, y activar la memoria personal o autobiográfica. Durante esta actividad se trabajará la orientación temporo-espacial y personal. Entre todos los participantes se indicará el año, el mes, el día de la semana y el día del mes. Después se abordará la orientación temporal (nombre del centro, ciudad, provincia y país). Finalmente, cada uno de ellos apuntará datos de orientación autobiográfica. Dependiendo de la programación de la sesión se abordarán, a través de breves tareas de reminiscencia, diferentes cuestiones como son: lugar de nacimiento, descripción de familiares, hechos vitales relevantes etc.

Psicoestimulación Cognitiva (Smartbrain): Dentro de los diversos tratamientos de aproximación cognitiva existentes, se decide aplicar las nuevas tecnologías y se implementa un plan de entrenamiento de las funciones cognoscitivas informatizado, realizado con ordenador. El motivo de la elección son las múltiples ventajas que aportan, puesto que son abordajes dinámicos, atractivos, flexibles, accesibles y además proporcionan al paciente un feedback inmediato de su ejecución. En concreto, Smartbrain es un sistema interactivo multimedia para la psicoestimulación cognitiva en pacientes con demencia Alzheimer en fase leve y moderada. Este instrumento permite una gran flexibilidad a la hora de programar ejercicios de psicoestimulación. Consta de un total de 12600 ejercicios distintos, que parten de siete categorías cognitivas; memoria, lenguaje, reconocimiento, atención, funciones ejecutivas, cálculo y orientación. Además, cada actividad tiene 15 niveles de dificultad. En nuestro caso, se crearán planes de trabajo individualizados en relación a las alteraciones que presenta el paciente y éstos se 
modificarán en función de su ejecución individual. Durante todo el proceso, el terapeuta aparte de tomar las decisiones acerca del tipo de intervención más adecuada y de establecer los objetivos, también supervisará continuamente los avances producidos y proporcionará apoyo para la adecuada participación en el programa.

Actividad grupal: Esta actividad grupal variará en cada sesión, teniendo en cuenta la programación semanal (Tabla 2). En general, el nivel de dificultad de las mismas es bajo, dado el nivel de escolaridad de los pacientes.

Cierre de la sesión: Al final de cada sesión se desarrollará un resumen que sintetice los resultados obtenidos por el grupo y los objetivos que se pretendían alcanzar. También se solicitará al paciente que realice un feedback sobre la sesión.

Tabla 2. Sesiones de la actividad grupal

\begin{tabular}{|c|c|}
\hline Sesiones de la actividad grupal & Objetivos \\
\hline 1. Dinámica de presentación & $\begin{array}{l}\text { Conocer al resto de compañeros y sus cualidades positivas. Estimular } \\
\text { un ambiente distendido. Ejercitar la memoria inmediata y la reciente. } \\
\text { Potenciar la creatividad }\end{array}$ \\
\hline 2. "Mesón Carmen" & $\begin{array}{c}\text { Optimizar los niveles de atención sobre el estímulo. Ejercitar el } \\
\text { cálculo. Favorecer los procesos atencionales y la concentración. } \\
\text { Potenciar los procesos amnésicos. }\end{array}$ \\
\hline $\begin{array}{l}\text { 3. Taller de aprendizaje y } \\
\text { memoria }\end{array}$ & $\begin{array}{c}\text { Favorecer la utilización de estrategias de aprendizaje. Ejercitar los } \\
\text { diferentes tipos y procesos de la memoria. Fomentar la } \\
\text { iniciativa y la fluidez verbal }\end{array}$ \\
\hline 4. Taller de reminiscencia & $\begin{array}{l}\text { Favorecer la evocación de recuerdos del pasado, conectándolos con } \\
\text { sucesos presentes. Estimular la propia identidad y la autoestima }\end{array}$ \\
\hline 5. "Pasapalabra" & $\begin{array}{l}\text { Maximizar las capacidades lingüísticas preservadas. Mantener y } \\
\text { estimular la comprensión verbal. Favorecer la incitación y la } \\
\text { fluidez verbal }\end{array}$ \\
\hline $\begin{array}{l}\text { 6. Taller de psicomotricidad y } \\
\text { praxias }\end{array}$ & $\begin{array}{c}\text { Optimizar la motricidad fina. Favorecer la producción de actos } \\
\text { motores voluntarios. Mejorar y/o mantener la coordinación y la } \\
\text { precisión de los movimientos }\end{array}$ \\
\hline $\begin{array}{l}\text { 7. Yincana de las } \\
\text { funciones ejecutivas }\end{array}$ & $\begin{array}{l}\text { Favorecer la reversibilidad y flexibilidad cognitiva. Ejercitar la } \\
\text { capacidad de planificación. Entrenar la capacidad de } \\
\text { secuenciación e inhibición de conductas inadecuadas }\end{array}$ \\
\hline $\begin{array}{l}\text { 8. Taller sobre el manejo } \\
\text { del dinero }\end{array}$ & $\begin{array}{c}\text { Reconocer el sistema monetario nacional, tanto las monedas } \\
\text { como los billetes. Entrenar el manejo del dinero. Promover el uso } \\
\text { del dinero en situaciones cotidianas. }\end{array}$ \\
\hline 9. Taller de memoria remota & $\begin{array}{l}\text { Facilitar el mantenimiento de la información adquirida años atrás. } \\
\text { Fomentar la fluidez verbal. Favorecer la evocación de recuerdos } \\
\text { pasados. Ejercitar el reconocimiento tanto visual como auditivo }\end{array}$ \\
\hline 10. "El mundo por los sentidos" & $\begin{array}{c}\text { Optimizar los niveles de atención y ejercitar la percepción a través } \\
\text { de material auditivo, gustativo y olfativo. Trabajar el } \\
\text { reconocimiento de estímulos }\end{array}$ \\
\hline 11. Aerobic con pelotas & $\begin{array}{l}\text { Mejorar la resistencia cardiovascular, la movilidad articular y la } \\
\text { coordinación }\end{array}$ \\
\hline $\begin{array}{l}\text { 12. Programa de entrenamiento } \\
\text { en actividades instrumentales de } \\
\text { la vida diaria }\end{array}$ & $\begin{array}{c}\text { Estimular la realización de las actividades instrumentales de la vida } \\
\text { diaria con el fin de mantener la capacidad } \\
\text { funcional }\end{array}$ \\
\hline
\end{tabular}

Las actividades propuestas comparten los siguientes objetivos generales: favorecer la comunicación y socialización entre los usuarios, y facilitar su expresión emocional. Todos los ejercicios se relacionarán por medio de ejemplos con situaciones cotidianas. El fin último de todas ellas es posibilitar el cambio real en la vida, es decir, la utilización al acontecer diario de cuanto se realice en las sesiones. 


\section{RESULTADOS}

Como puede observarse en las figuras 1 y 2, la evolución de los pacientes no es homogénea. A nivel cognitivo, se objetivan discretas mejorías. La puntuación obtenida en el test MEC oscila entre 30 y 20 puntos, lo que supone un deterioro leve- moderado. El $50 \%$ de los pacientes que participaron en el programa obtienen la misma puntuación que al inicio, el 37,5\% obtuvo una mejoría respecto a la puntuación inicial y únicamente el $12,5 \%$ empeoró respecto a la puntuación base.

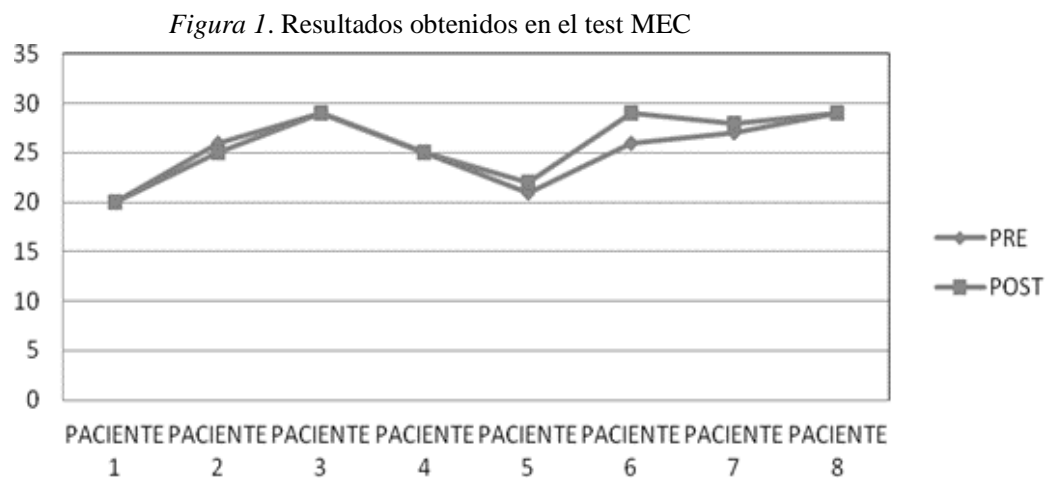

En relación a la calidad, el $50 \%$ de los pacientes mejoran respecto a la calificación inicial, el 12,5\% mantiene la misma nota, sin embargo, 37,5\% empeoran. A pesar de que la intervención no produjo mejorías significativas en las variables medidas, los pacientes referían una mejoría clínica principalmente a nivel funcional.

Figura 2. Resultados obtenidos en la escala QOL-AD

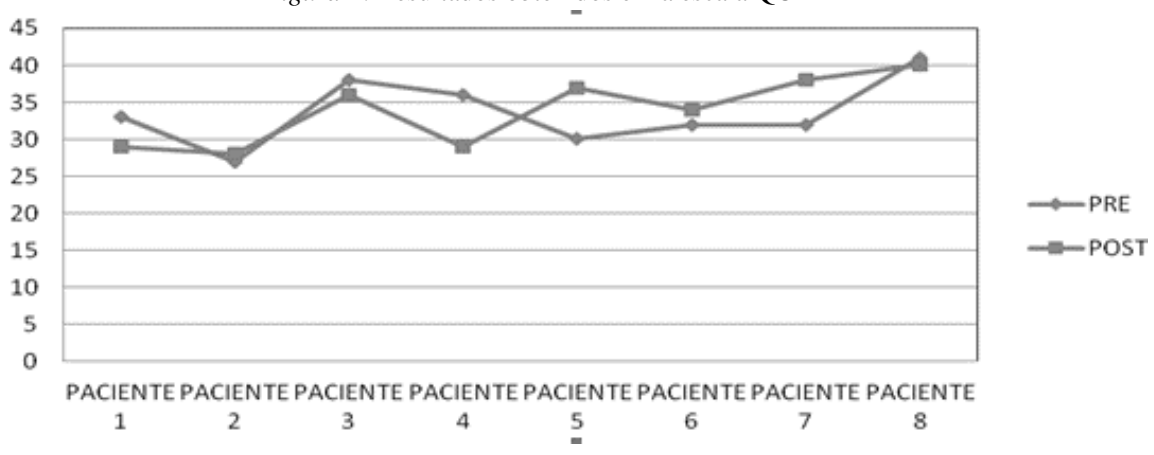




\section{DISCUSIÓN Y CONCLUSIONES}

La EA se define por un cuadro de demencia, es decir, por un proceso progresivo de deterioro cognitivo y funcional de inicio insidioso (Tárraga, 1991). A nivel cognitivo, la alteración principal se objetiva en la memoria episódica verbal a la que posteriormente se asociaran otras afectaciones cognitivas. En el área funcional, se produce un declive progresivo en la actividad funcional del paciente, por lo que tanto las AVD como su calidad de vida, se irán mermando.

El tratamiento de la demencia consiguientemente, requiere un tratamiento integral. La propuesta de intervención que se plantea es un programa de psicoestimulación integral que se compone de tres estrategias terapéuticas no farmacológicas; terapia de orientación a la realidad, la psicoestimulación cognitiva y la actividad grupal, con el objetivo de mantener el estado cognitivo, mejorar la autonomía de los pacientes y de este modo, mejorar su calidad de vida y la de sus familiares.

En la revisión sistemática realizada por Olazarán et al. (2010) se confirma la eficacia de las TNF en la EA, concretamente las intervenciones multicomponentes obtienen recomendación grado B, principalmente en las áreas de cognición, AVD, conducta, estado de ánimo y calidad de vida. Los resultados obtenidos en nuestro estudio, a pesar de vislumbrar resultados esperanzadores tanto en el área cognitiva como en calidad de vida, éstos no son estadísticamente significativos, si bien los pacientes si referían una mejoría clínica a nivel funcional.

Podemos concluir que nuestro estudio ha de ser considerado como un estudio preliminar con resultados prometedores, siendo necesario, por un lado, ampliar la muestra de estudio y por otro, implementar un programa de mayor continuidad e intensidad para poder obtener unos resultados significativos y demostrar así la eficacia de la psicoestimulación integral en pacientes con diagnóstico de EA.

Las líneas futuras de investigación pueden dirigirse, por un lado, a mejorar la metodología de la investigación del presente estudio con el objetivo de determinar de forma específica la eficacia de cada componente y así como, los efectos diferenciales de los mismos. Por otro lado, sería necesario ampliar el tamaño muestral para replicar el estudio, incluyendo grupos control con pacientes con deterioro cognitivo que únicamente reciban los cuidados habituales y otro grupo de sujetos sin deterioro cognitivo. Además, sería recomendable implementar un programa de mayor continuidad en el tiempo y de mayor frecuencia semanal, así como ampliar el seguimiento, realizando una reevaluación en periodos más prolongados de tiempo. Por último, se debería valorar la repercusión de la intervención en los cuidadores y familiares.

Resulta de gran importancia continuar aplicando programas de TNF en pacientes afectados de EA, así como estudiar su eficacia, ya que, a pesar de los beneficios derivados 
de algunas de estas intervenciones, su presencia en los programas de tratamiento es, en general, todavía menor de la esperada.

\section{REFERENCIAS}

Alberca, R., y López, P.S. (2011). Enfermedad de Alzheimer y otras demencias. Madrid, España: Editorial Médica Panamericana.

Francés, I., Barandiarán, M., Marcellán, T., y Moreno, L. (2003). Estimulación psicocognoscitiva en las demencias. Anales del Sistema Sanitario de Navarra, 26(3), 383-403.

Franco, M.A., y Orihuela, T. (1998). Programa AIRE: Sistema multimedia de evaluación y entrenamiento cerebral. Valladolid, España: Edlntras.

García, J.J., y Carro, J. (2011). Programa de actuación cognitiva integral en demencias (PACID): centro de referencia estatal de atención a personas con enfermedad de Alzheimer y otras demencias. Madrid, España: IMSERSO.

Hoe, J., Katona, C., Roch, B., y Livingston, G. (2005). Use of the qol-ad for measuring quality of life in people with severe dementia--the laser-ad study. Age and Ageing, 34(2), 130-135.

Lobo, A., Saz, P., Marcos, G., Día, J.L., de la Cámara, C., Ventura, T., y Aznar, S. (1999). Revalidación y normalización del Mini-Examen Cognoscitivo (primera versión en castellano del Mini-Mental Status Examination) en la población general geriátrica. Medicina Clínica, 112(20), 767-774.

Olazarán, J., Muñiz, R., Reisberg, B., Peña, J., Del Ser, T., Cruz, A. J., y Galiano, M. (2004). Benefits of cognitive-motor intervention in MCI and mild to moderate Alzheimer disease. Neurology, 63(12), 2348-2353.

Olazarán, J., Poveda, S., Martínez, U., y Muñiz, R. (2007). Estimulación cognitiva y tratamiento no farmacológico. En: J.M. Martínez, y C. Pardo (Eds), Alzheimer 2007: recapitulación y perspectivas (pp. 91-102). Madrid: Aula Médica.

Olazarán, J., Reisberg, B., Clare, L., Cruz, I., Peña, J., del Ser, T., y Muñiz, R. (2010). Eficacia de las terapias no farmacológicas en la enfermedad de Alzheimer: una revisión sistemática. Dementia and Geriatric Cognitive Disorders, 30(2), 161-178.

Peña, J. (2007). Neurología de la conducta y neuropsicología. Madrid, España: Editorial Médica Panamericana.

Tárraga, L. (1991). Programa de Psicoestimulación Integral (PPI). Tratamientos de psicoestimulación. En R. Fernández, y J. Nicolás (Eds), Libro blanco sobre la Enfermedad de Alzheimer, Volumen I (pp. 112-121). Madrid: Caja Madrid Obra Social.

Villar, A., Molinuevo, J.L., y Gómez, T. (2004). Enfermedad de Alzheimer. Jano, 67, 1537-1541.

Virués, J., de Pedro, J., Vega, S., Seijo, M., Saz, P., Rodríguez, F., y Martínez-Martín, P. (2011). Prevalence and European comparison of dementia in a $\geq 75$-year-old composite population in Spain. Acta Neurologica Scandinavica, 123(5), 316-324.

Recibido: 14 de noviembre de 2019

Recepción Modificaciones: 23 de diciembre de 2019

Aceptado: 26 de diciembre de 2019 